7. Нестеренко А. Н., Химич А. Н., Яковлев М. Ф. Некоторые вопросы решения систем нелинейных уравнений на многопроцессорных вычислительных системах с распределенной памятью. Вестник компьютерных и информационных технологий. М., 2006. № 10. С. 54-56.

\title{
SOLVING OF THE SYSTEMS OF NON-LINEAR EQUATIONS ON COMPUTERS WITH PARALLEL ORGANIZATION OF CALCULATIONS
}

The methodology for the solving of non-linear systems with sparse Jacobi matrices on parallel computers is proposed, which uses a multilevel parallel computing model, structural regularization and decomposition of sparse data for reducing system's matrix to the block-sparse form, high-performance block and block-cyclic algorithms for solving systems of linear equations.

Key words: systems of non-linear equations, sparse Jacobi matrices, MIMD-architecture computers, hybrid-architecture computers, structural regularization of the sparse data, decomposition of the sparse data.

Одержано 15.02.2019

УДК 519.9

DOI: $10.32626 / 2308-5878.2019-19.91-97$

О. П. Нечуйвітер, д-р фіз.-мат. наук,

Г. В. Каргапольцева, здобувач,

К. В. Дараган, аспірантка

Українська інженерно-педагогічна академія, м. Харків

\section{ОПТИМАЛЬНА ЗА ПОРЯДКОМ ТОЧНОСТІ КУБАТУРНА ФОРМУЛА НАБЛИЖЕНОГО ОБЧИСЛЕННЯ ПОДВІЙНОГО ІНТЕГРАЛУ ВІД ШВИДКООСЦИЛЮЮЧИХ ФУНКЦІЙ ЗАГАЛЬНОГО ВИДУ}

\begin{abstract}
Розглядається оптимальна за порядком точності кубатурна формула наближеного обчислення подвійного інтегралу від швидкоосцилюючих функцій загального виду на класі диференційовних функцій у випадку, коли інформація про функції задана їх слідами на відповідних лініях.
\end{abstract}

Ключові слова: кубатурна формули, інтеграли від швидкоосцилюючих функиій, клас диференційовних функиій.

Вступ. Задача наближеного обчислення інтегралів від швидкоосцилюючих функцій двох змінних загального виду

$$
I(f, g, \omega)=\int_{0}^{1} \int_{0}^{1} f(x, y) \mathrm{e}^{i \omega g(\mathrm{x}, \mathrm{y})} d x d y,
$$


має як класичне розв'язання [1], так і у випадку різних інформаційних операторів [2, 3]. Однак, не дослідженим залишилось питання побудови оптимальних за порядком точності кубатурних формул, у випадку, коли інформація про $f(x, y)$ та $g(x, y)$ задана відповідними їх слідами на лініях. Дана стаття присвячена актуальному питанню: побудові оптимальної за порядком точності кубатурної формули обчислення інтегралу виду (1) на класі диференційовних функцій.

Оптимальна за порядком точності кубатурна формула. Припустимо, що $f(x, y) \in F, g(x, y) \in G, F, G$ - множини функцій, визначених в області $[a, b] \times[a, b]$. Позначимо $L_{N}$ множину всіх квадратурних формул $l_{N}(f, g)$, що використовують інформацію про значення функцій $f(x, y)$ та $g(x, y)$ не більше ніж на $N$ лініях. Введемо величини $R_{N}\left(f, g, \omega, l_{N}\right)=\left|I(f, g, \omega)-l_{N}(f, g)\right|$,

$$
\begin{aligned}
R_{N}\left(F, G, \omega, l_{N}\right) & =\sup _{f \in F, g \in G} R_{N}\left(f, g, \omega, l_{N}\right), \\
R_{N}(F, G, \omega) & =\inf _{l_{N} \in L_{N}} R_{N}\left(F, G, \omega, l_{N}\right) .
\end{aligned}
$$

Кубатурну формулу $l_{N}^{*}(f, g)$, на якій досягається $R_{N}(F, G, \omega)$, будемо називати оптимальною за точністю кубатурною формулою. Якщо $R_{N}\left(F, G, \omega, \bar{l}_{N}\right) \leq R_{N}(F, G, \omega)+\eta, \eta>0$, то $\bar{l}_{N}$ називається оптимальною за точністю формулою обчислення $I(f, g, \omega)$ з точністю до $\eta$. Якщо $\eta=o\left(R_{N}\right)$ або $\eta=O\left(R_{N}\right)$, то $\bar{l}_{N}$ називається асимптотично оптимальною або оптимальною за порядком точності.

Розглянемо $H^{2, r}(M, M)$ - клас дійсних функцій, визначених на $G=[0,1]^{2}$ і таких, що частинні похідні порядку $r$ по змінній $x$ та $y$ обмежені, тобто

$$
\left|f^{(r, 0)}(x, y)\right| \leq M,\left|f^{(0, r)}(x, y)\right| \leq M, r \neq 0,\left|f^{(r, r)}(x, y)\right| \leq M, \quad r \geq 0 .
$$

Теорема 1 [3]. Нехай $f(x, y), g(x, y), \in H^{2, r}(M, M)$, функції $f(x, y), g(\mathrm{x}, \mathrm{y})$ задані слідами на відповідних системах взаємно перпендикулярних прямих в області $G=[0,1]^{2}$, тоді

$$
R_{N}\left(H^{2, r}(\mathrm{M}, M), H^{2, r}(M, M), \omega\right) \geq K \max \left\{\frac{1}{\ell^{2 r}}, \min \left\{1, \frac{|\omega|}{\ell^{2 r}}\right\}\right\} .
$$


Під слідом функції $f(x, y)$ на лініях $x_{k}=k \Delta_{1}-\Delta_{1} / 2$, $y_{j}=j \Delta_{1}-\Delta_{1} / 2, k, j=\overline{1, \ell_{1}}, \Delta_{1}=1 / \ell_{1}$ розуміємо відповідно функції однієї змінної $f\left(x_{k}, y\right), 0 \leq y \leq 1, f\left(x, y_{j}\right), 0 \leq x \leq 1$. Під слідом функції $g(x, y)$ на лініях $x_{p}=p \Delta_{2}-\Delta_{2} / 2, \quad y_{s}=s \Delta_{2}-\Delta_{2} / 2$, $p, s=\overline{1, \ell_{2}}, \Delta_{2}=1 / \ell_{2}$ розуміємо відповідно функції однієї змінної $g\left(x_{p}, y\right), 0 \leq y \leq 1, g\left(x, y_{s}\right), 0 \leq x \leq 1$.

Нехай

$$
\begin{aligned}
& h 1_{0 k}(x)=\left\{\begin{array}{l}
1, x \in X 1_{k}, \\
0, x \notin X 1_{k},
\end{array} \quad k=\overline{1, \ell_{1}}, \quad H 1_{0 j}(y)=\left\{\begin{array}{l}
1, y \in Y 1_{j}, \\
0, y \notin Y 1_{j},
\end{array} \quad j=\overline{1, \ell_{1}},\right.\right. \\
& X 1_{k}=\left[x_{k-1 / 2}, x_{k+1 / 2}\right], Y 1_{j}=\left[y_{j-1 / 2}, y_{j+1 / 2}\right], \\
& x_{k}=k \Delta_{1}-\Delta_{1} / 2, \quad y_{j}=j \Delta_{1}-\Delta_{1} / 2, k, j=\overline{1, \ell_{1}}, \Delta_{1}=1 / \ell_{1} \text {, } \\
& h 2_{0 p}(x)=\left\{\begin{array}{l}
1, x \in X 2_{p}, \\
0, x \notin X 2_{p},
\end{array} \quad p=\overline{1, \ell_{1}}, \quad H 2_{0 j}(y)=\left\{\begin{array}{l}
1, y \in Y 2_{s}, \\
0, y \notin Y 2_{s},
\end{array} \quad s=\overline{1, \ell_{1}},\right.\right. \\
& X 1_{p}=\left[x_{p-1 / 2}, x_{p+1 / 2}\right], Y 1_{s}=\left[y_{s-1 / 2}, y_{s+1 / 2}\right], \\
& x_{p}=p \Delta_{2}-\Delta_{2} / 2, y_{s}=s \Delta_{2}-\Delta_{2} / 2, \mathrm{p}, \mathrm{s}=\overline{1, \ell_{2}}, \Delta_{2}=1 / \ell_{2} \text {. }
\end{aligned}
$$

Розглянемо оператори

$$
\begin{aligned}
J_{\ell_{1}}(x, y)= & \sum_{k=1}^{\ell_{1}} f\left(x_{k}, y\right) h 1_{0 k}(x)+\sum_{j=1}^{\ell_{1}} f\left(x, y_{j}\right) H 1_{0 j}(y)- \\
& -\sum_{k=1}^{\ell_{1}} \sum_{j=1}^{\ell_{1}} f\left(x_{k}, y_{j}\right) h 1_{0 k}(x) H 1_{0 j}(y), \\
O_{\ell_{2}}(x, y)= & \sum_{p=1}^{\ell_{2}} g\left(x_{p}, y\right) h 2_{0 p}(x)+\sum_{s=1}^{\ell_{2}} g\left(x, y_{s}\right) H 2_{0 s}(y)- \\
& -\sum_{p=1}^{\ell_{2}} \sum_{s=1}^{\ell_{2}} g\left(x_{p}, y_{s}\right) h 2_{0 p}(x) H 2_{0 s}(y) .
\end{aligned}
$$

Кубатурна формула

$$
\Phi^{2}(\omega)=\int_{0}^{1} \int_{0}^{1} J_{\ell_{1}}(x, y) \mathrm{e}^{i \omega O_{\ell_{2}}(x, y)} d x d y
$$

пропонується для наближеного обчислення інтегралу

$$
I^{2}(\omega)=I(f, g, \omega) .
$$


Теорема 2. Нехай $f(x, y), g(x, y) \in H^{2,1}(M, M)$, функції $f(x, y), g(x, y)$ задані слідами $f\left(x_{k}, y\right), k=\overline{1, \ell_{1}}, f\left(x, y_{j}\right), j=\overline{1, \ell_{1}}$; $g\left(x_{p}, y\right), p=\overline{1, \ell_{2}}, \quad g\left(x, y_{s}\right), s=\overline{1, \ell_{2}}$ на $N=2 \ell_{1}+2 \ell_{2}$ взаємно перпендикулярних прямих в області $G=[0,1]^{2}$. Тоді кубатурна формула $\Phi^{2}(\omega) \epsilon$ оптимальною за порядком точності, для якої справедливі наступні оцінки:

$$
\begin{gathered}
\rho\left(I^{2}(\omega), \Phi^{2}(\omega)\right)= \\
=\left|\int_{0}^{1} \int_{0}^{1} f(x, y) \mathrm{e}^{i \omega g(\mathrm{x}, \mathrm{y})} d x d y-\int_{0}^{1} \int_{0}^{1} J_{\ell_{1}}(x, y) \mathrm{e}^{i \omega O_{\ell_{2}}(x, y)} d x d y\right| \leq \\
\leq \frac{M}{16} \frac{1}{\ell_{1}^{2}}+M \min \left(2 ; \frac{M \omega}{16} \frac{1}{\ell_{2}^{2}}\right),
\end{gathered}
$$

та

$$
R_{N}\left(H^{2,1}(\mathrm{M}, M), H^{2,1}(M, M), \omega\right) \geq K \max \left\{\frac{1}{\ell^{2}}, \min \left\{1, \frac{|\omega|}{\ell^{2}}\right\}\right\}
$$

при $\ell_{1}=\ell_{2}=\ell$.

\section{Доведення. Оцінка}

$$
R_{N}\left(H^{2,1}(\mathrm{M}, M), H^{2,1}(M, M), \omega\right) \geq K \max \left\{\frac{1}{\ell^{2}}, \min \left\{1, \frac{|\omega|}{\ell^{2}}\right\}\right\}
$$

отримується з теореми 1 при $r=1$.

В роботі [2] показано, що

$$
\begin{aligned}
& \rho\left(I^{2}(\omega), \Phi^{2}(\omega)\right)= \\
& =\left|\int_{0}^{1} \int_{0}^{1} f(x, y) \mathrm{e}^{i \omega g(\mathrm{x}, \mathrm{y})} d x d y-\int_{0}^{1} \int_{0}^{1} J_{\ell_{1}}(x, y) \mathrm{e}^{i \omega O_{\ell_{2}}(x, y)} d x d y\right| \leq \\
& \leq \int_{0}^{1} \int_{0}^{1}\left|f(x, y)-J_{\ell_{1}}(x, y)\right| d x d y+\int_{0}^{1} \int_{0}^{1}|f(x, y)|\left|\mathrm{e}^{i \omega g(\mathrm{x}, \mathrm{y})}-\mathrm{e}^{i \omega O_{\ell_{2}}(x, y)}\right| d x d y \leq \\
& \leq \sum_{k=1}^{\ell_{1}} \sum_{j=1}^{\ell_{1}} \int_{x_{k-\frac{1}{2}}}^{x_{k+\frac{1}{2}}} \int_{j=\frac{1}{2}}^{y}\left|\int_{x_{k}}^{y} \int_{y_{j}}^{y} f^{(1,1)}(\xi, \eta) d \xi d \eta\right| d x d y+
\end{aligned}
$$




$$
\begin{gathered}
+2 M \sum_{p=1}^{\ell_{2}} \sum_{s=1}^{\ell_{2}} \int_{x_{p-\frac{1}{2}}}^{x_{p+\frac{1}{2}}} \int_{y_{s-\frac{1}{2}}}^{y} \min \left(1 ; \frac{\omega}{2}\left|\int_{x_{p}}^{x} \int_{y_{s}}^{y} g^{(1,1)}(\xi, \eta) d \xi d \eta\right|\right) d x d y \leq \\
\leq \frac{M}{16} \Delta_{1}^{2}+2 M \min \left(\ell_{2}^{2} \Delta_{2}^{2}, \frac{M \omega}{2} \ell_{2}{ }^{2} \frac{\Delta_{2}{ }^{2}}{4} \frac{\Delta_{2}^{2}}{4}\right)= \\
=\frac{M}{16} \Delta_{1}^{2}+M \min \left(2 ; \frac{M \omega}{16} \Delta_{2}^{2}\right)=\frac{M}{16} \frac{1}{\ell_{1}^{2}}+M \min \left(2 ; \frac{M \omega}{16} \frac{1}{\ell_{2}^{2}}\right) .
\end{gathered}
$$

При $\ell_{1}=\ell_{2}=\ell$, маємо

$$
\rho\left(I^{2}(\omega), \Phi^{2}(\omega)\right) \leq \frac{M}{16} \frac{1}{\ell^{2}}+M \min \left(2 ; \frac{M \omega}{16} \frac{1}{\ell^{2}}\right) \leq C \max \left(\frac{1}{\ell^{2}}, \min \left(1 ; \frac{|\omega|}{\ell^{2}}\right)\right) .
$$

Порівнюючи оцінки зверху та знизу, робимо висновок про оптимальність за порядком точності кубатурної формули. Теорема доведена.

Теорема 3. Нехай для $f(x, y), g(x, y)$ виконуються умови теореми 2. Тоді для кубатурної формули

$$
\Phi_{s}^{2}(\omega)=\int_{0}^{1} \int_{0}^{1} J_{\ell_{1}}(x, y) \sin \left(\omega O_{\ell_{2}}(x, y)\right) d x d y
$$

наближеного обчислення

$$
I_{s}^{2}(\omega)=\int_{0}^{1} \int_{0}^{1} f(x, y) \sin (\omega g(\mathrm{x}, \mathrm{y})) d x d y
$$

справедлива наступна оцінка:

$$
\rho\left(I_{s}^{2}(\omega), \Phi_{s}^{2}(\omega)\right) \leq \frac{M}{16} \frac{1}{\ell_{1}^{2}}+M \min \left(2 ; \frac{M \omega}{16} \frac{1}{\ell_{2}^{2}}\right) .
$$

Доведення. Аналогічно доведенню теореми 2,

$$
\begin{aligned}
& \rho\left(I_{s}^{2}(\omega), \Phi_{s}^{2}(\omega)\right) \leq \int_{0}^{1} \int_{0}^{1}\left|f(x, y)-J_{\ell_{1}}(x, y)\right| d x d y+ \\
& +\int_{0}^{1} \int_{0}^{1}|f(x, y)|\left|\sin (\omega g(x, y))-\sin \left(\omega O_{\ell_{2}}(x, y)\right)\right| d x d y \leq \\
& \quad \leq \int_{0}^{1} \int_{0}^{1}\left|f(x, y)-J_{\ell_{1}}(x, y)\right| d x d y+ \\
& +\int_{0}^{1} \int_{0}^{1}|f(x, y)| 2 \sin \frac{\omega \mathrm{g}(\mathrm{x}, \mathrm{y})-\omega O_{\ell_{2}}(x, y)}{2} \cos \frac{\omega \mathrm{g}(\mathrm{x}, \mathrm{y})+\omega O_{\ell_{2}}(x, y) \mid}{2} \mid d x d y \leq
\end{aligned}
$$




$$
\begin{aligned}
& \leq \sum_{k=1}^{\ell_{1}} \sum_{j=1}^{\ell_{1}} \int_{x_{k-\frac{1}{2}}}^{x_{k+\frac{1}{2}}} \int_{y_{j-\frac{1}{2}}}^{y+\frac{1}{2}}\left|\int_{x_{k}}^{x} \int_{y_{j}}^{y} f^{(1,1)}(\xi, \eta) d \xi d \eta\right| d x d y+ \\
& +2 M \sum_{p=1}^{\ell_{2}} \sum_{s=1}^{\ell_{2}} \int_{x}^{x+\frac{1}{2}} \int_{y_{s-\frac{1}{2}}}^{y} \min \left(1 ; \frac{\omega}{2}\left|\int_{x_{p}}^{x} \int_{y_{s}}^{y} g^{(1,1)}(\xi, \eta) d \xi d \eta\right|\right) d x d y \leq \\
& \leq \frac{M}{16} \Delta_{1}^{2}+M \min \left(2 ; \frac{M \omega}{16} \Delta_{2}^{2}\right)=\frac{M}{16} \frac{1}{\ell_{1}^{2}}+M \min \left(2 ; \frac{M \omega}{16} \frac{1}{\ell_{2}^{2}}\right) \text {. }
\end{aligned}
$$

Чисельні результати. Обчислимо $I_{s}^{2}(\omega)$ за формулою $\Phi_{s}^{2}(\omega)$ (таблиця) у випадку, коли $f(x, y)=\sin (x+y), g(x, y)=\cos (x+y)$ в MathCad 15.0. Точні значення інтегралів:

$$
I_{s}^{2}(2 \pi)=0.062699216073162, I_{s}^{2}(5 \pi)=0.022780463640219 .
$$

Нехай $\varepsilon_{e x}=\left|I_{s}^{2}(\omega)-\Phi_{s}^{2}(\omega)\right|$. Покажемо, що

$$
\varepsilon_{e x} \leq \varepsilon_{t h}, \quad \varepsilon_{t h}=\frac{M}{16} \frac{1}{\ell_{1}^{2}}+M \min \left(2 ; \frac{M \omega}{16} \frac{1}{\ell_{2}{ }^{2}}\right) \text {. }
$$

Таблиця

Обчислення $I_{s}^{2}(\omega)$ за формулою $\Phi_{s}^{2}(\omega)$

\begin{tabular}{|c|c|c|c|c|c|}
\hline$\omega$ & $\ell_{1}$ & $\ell_{2}$ & $\Phi_{s}^{2}(\omega)$ & $\varepsilon_{\text {ex }}$ & $\varepsilon_{\text {th }}$ \\
\hline $2 \pi$ & 4 & 4 & 0.062432583948326 & $2.6 \cdot 10^{-4}$ & $2.8 \cdot 10^{-2}$ \\
\hline $2 \pi$ & 7 & 7 & 0.062683978467995 & $1.5 \cdot 10^{-5}$ & $9.2 \cdot 10^{-3}$ \\
\hline $5 \pi$ & 6 & 4 & 0.022786668787906 & $6.2 \cdot 10^{-6}$ & $2.9 \cdot 10^{-2}$ \\
\hline $5 \pi$ & 10 & 4 & 0.022808425368659 & $2.7 \cdot 10^{-5}$ & $6.1 \cdot 10^{-2}$ \\
\hline $5 \pi$ & 10 & 10 & 0.02277048162594 & $9.9 \cdot 10^{-6}$ & $1.04 \cdot 10^{-2}$ \\
\hline
\end{tabular}

Висновки. Розглядається кубатурна формула наближеного обчислення подвійних інтегралів від швидкоосцилюючих функцій загального виду у випадку, коли інформація про функції задана їх слідами на відповідних лініях. Доведена оптимальність за порядком точності запропонованої кубатурної формули на класі диференційовних функцій. Чисельний експеримент підтвердив теоретичні твердження.

\section{Список використаних джерел:}

1. Сергієнко І. В., Задірака В. К., Литвин О. М., Мельникова С. С., Нечуйвітер О. П. Оптимальні алгоритми обчислення інтегралів від швидкоосци- 
люючих функцій та їх застосування : у 2 т. Т. 1. Алгоритми : [монографія]. Київ : Наук. думка, 2011. 447 с.

2. Lytvyn O. M., Nechuiviter O., Pershyna Yu., Mezhuyev V. Input Information in the Approximate Calculation of Two-Dimensional Integral from Highly Oscillating Functions (Irregular Case). Recent Developments in Data Science and Intelligent Analysis of Information. Proceedings of the XVIII International Conference on Data Science and Intelligent Analysis of Information, June 4-7, 2018. Kyiv, Ukraine. P. 365-373.

3. Mezhuyev V., Lytvyn O. M., Nechuiviter O., Pershyna Yu., Lytvyn O. O., Keita K. Cubature formula for approximate calculation of integrals of twodimensional irregular highly oscillating functions. U.P.B. Sci. Bull., Series A. Vol. 80, Iss. 3. 2018. P. 169-182.

4. Нечуйвітер О. П., Кейта К. В. Оптимальне інтегрування двовимірних швидкоосцилюючих функцій загального виду. Математичне та комп'ютерне моделювання. Сер. Фіз.-мат. науки : зб. наук. пр. Кам'янець-Подільський : Кам'янець-Подільський нац. ун-т ім. Івана Огієнка, 2017. Вип. 15. С. 139-144.

\section{THE OPTIMAL BY THE ORDER OF EXACTNESS CUBATURE FORMULA FOR CALCULATION OF TWO-DIMEN- SIONAL INTEGRAL FROM HIGHLY OSCILLATING FUNCTIONS OF GENERAL VIEW}

The paper is devoted to the optimal by the order of exactness cubature formula for calculation of two-dimensional integral from highly oscillating functions of general view in case when the information about functions is a set of lines.

Key words: cubature formula, integral from highly oscillating function, class of differentiable functions.

Одержано 24.01.2019 